\title{
Fluorometric calcium measurement
}

\author{
J.S. Hall, K.A. Korkidis and D.L. Maskevich
}

\section{Measuring the fluorescence of dyes specific for calcium and other cations allows the elucidation of cellular mechanisms such as neurotransmission, muscle contraction and cardiovascular regulation.}

A MAJOR focal point of biomedical research over the past decade has been the study of the basic role of $\mathrm{Ca}^{2+}$ in physiological events such as neurotransmission, muscle contraction, tumour growth and cardiovascular regulation'. Work in this area has been advanced greatly by the ability to measure $\mathrm{Ca}^{2+}$ in living cells using fluorometric techniques that employ fluorogenic dyes or "probes" with known $\mathrm{Ca}^{2+}$ affinity together with specialized commercial instruments which allow the optimal use of these probes ${ }^{2.3}$.

Fluorometry offers distinct advantages over $\mathrm{Ca}^{2+}$ measurements based upon radioactive isotopes, micro-electrodes or photoproteins. Radioactive material presents special handling problems, and micro-electrodes cause cellular damage, measure only local responses, and are limited to use in larger cells. Similarly, calcium-specific photoproteins are slow to disperse and must be introduced by injection, which ruptures cell membranes.

Cation analysis using instruments designed for research with fluorescent probes avoids these drawbacks. Fluorometric analysis is the least biologically disruptive of the techniques thus far devised for intracellular $\mathrm{Ca}^{2+}$ measurement. Fluorometry also permits much faster tracking of cation concentration changes, even at millisecond sampling rates. The technique is not confined exclusively to $\mathrm{Ca}^{2+}$; there are ion-specific fluorescent probes for the measurement of $p \mathrm{H}$ and other cations in healthy, living cells ${ }^{4.5}$. A single instrument system may be utilized for the measurement of cations in macroenvironments involving cell populations in suspension or for the micro-measurement of cations in individual cells on a microscope stage.

The most accurate fluorescent cation probes have distinctive dual-excitation or dual-emission characteristics: the fluorescence spectra of these probes change markedly depending upon whether they are free or bound to a cation. Experimental conditions determine the two optimum excitation or emission wavelengths for a specific measurement. At each wavelength there will be a pronounced fluorescence signal corresponding to the free or bound state, and a signal of significantly lower intensity for the opposite state. It is this differential fluorescence intensity that establishes the ratio that enables the measurement of the actual concentration of a cation. Ratioing

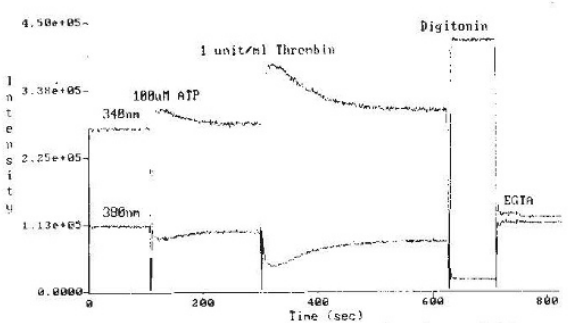

Fig. 1 Fluorescence data acquired at $340 \mathrm{~nm}$ and $380 \mathrm{~nm}$ from human umbilical vein endothelial cells treated with fura- 2 .

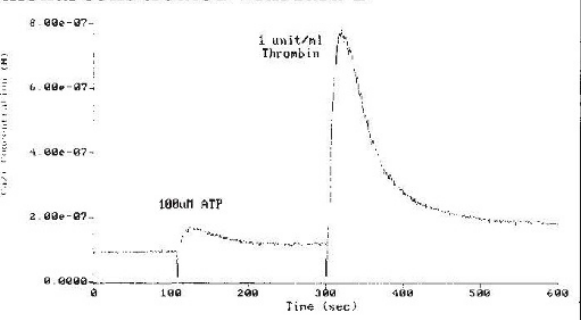

Fig. 2 Changes in the concentration of cytosolic free $\mathrm{Ca}^{2+}$ calculated directly from the fluorescence data in Fig. 1.

the two fluorescence signals from a sample also corrects for instrument artefacts, cellto-cell variations in probe concentration, and reduced fluorescence from photobleaching.

Figures 1 and 2 illustrate the $\mathrm{Ca}^{2+}$ flux in human umbilical vein endothelial cells resulting from the sequential addition of the agonists ATP and thrombin. For these measurements, the cells were treated with the dual-excitation probe fura- 2 . The lysing agent digitonin and the calcium chelator EGTA were used to set the range over which the concentration measurements were made. Figure 1 is a temporal plot of the fluorescence intensity of the sample at excitation wavelengths of 340 and $380 \mathrm{~nm}$. Figure 2 shows the absolute change in the concentration of cytosolic free calcium directly calculated from the data in Fig. 1.

A series of experiments on the contraction mechanism in cultured vascular smooth muscle cells (VSMC) obtained from the aortae of rats by researchers affiliated with Brigham and Women's Hospital and Harvard Medical School demonstrates the sensitivity and flexibility of fluorometric cation measurement ${ }^{6.7}$. They show that vasoconstrictor agonists induce rapid changes in intracellular $\mathrm{Ca}^{2+}$ and $p \mathrm{H}$, and that these ionic events are mimicked by platelet-derived growth factor (PDGF), a potent VSMC mitogen. The fluorescence techniques used in the experiment revealed that $\mathrm{Ca}^{2+}$ is mobi- lized from an intracellular storage site, while a rise in $p \mathrm{H}$ is mediated by the activation of $\mathrm{Na}^{+} / \mathrm{H}^{+}$exchange.

Although fluorometric cation measurement with dual-excitation probes has become the technique of choice for many researchers, the continuing evolution of hardware and methodology promise even greater experimental utility. Commercial hardware systems are available that incorporate two emission channels for macro- and micro-measurements with dual-emission dyes such as the $\mathrm{Ca}^{2+}$ probe indo- 1 . The recent addition of dedicated digital image processing equipment to existing cation measurement systems gives fluorescence analysis an entirely new dimension by enabling the collection of spatial data about cation migration and concentration.

Without imaging capability, fluorometric analysis of micro-samples can measure only changes in average intracellular fluorescence intensity over time. Image processing can pinpoint the location of site-specific biochemical events within a cell and distinguish the heterogeneous behaviour of cells in an apparently uniform sample. Similarly, fluorescence image processing makes it feasible to monitor ionic changes associated with cell-to-cell communication ${ }^{8}$.

The utility of fluorometric cation measurements is being expanded through the development of a new generation of ion-specific probes. In addition to new specialized calcium dyes, there are probes which promise considerable potential for the measurement of free magnesium, potassium and sodium ions ${ }^{9}$ in many types of living cells, including platelets, renal cells, osteoblasts and myocardial cells.

Jonathan Hall, Katherine Korkidis and Dean Maskevich are at Spex Industries, Inc., 3880 Park Avenue, Edison, New Jersey 08820, USA. For more information, fill in reader service number 100.

1. Kim, D., Okada, A. \& Smith. T.W. Circulation Res, 61, 29 (1987).

2. Grynkiewicz, G., Poenie, M. \& Tsien. R.Y. J. biol. Chem 260, 3440-3450 (1985).

3. Tsien, R.Y. Nature 290, 527-537 (1981)

4. Paradiso, A.M., Tsien, R.Y. \& Machen. T.E. Nature 325, $447-450(1987)$.

. Paradiso, A.M., Tsien, R.Y. \& Machen, T.E. Proc. natn Acad. Sci. USA 81, 7436-7440 (1985)

. Berk, B.C. et al. J. biol. Chem. 262, 5057-5064 (1987)

7. Berk, B.C., Brock, I.A., Gimbrone, Jr.. M.A.\& Alexander. R.W. J. biol Chem. 262, 5065-5072 (1987)

8. Tsien, R.Y.\& Poenie, M. Trends biochem. Sci. 11, 450-455 (1986),

9. Minta, A., Harootuniun, A.T., Kao, J.P. \& Tsien, R.Y 1987 Annual Meeting of the American Society for Cell Biology. Abstr. No. 492 (1987). 